\title{
Analysis of Light Leakage in Liquid-Crystal Devices with In-Plane Switching Electrodes
}

\author{
Hideo Kawano* Student Member \\ Atsushi Matsumoto* Non-member \\ Johji Hirano* Non-member \\ Tanroku Miyoshi* Non-member
}

\begin{abstract}
To realize wider viewing angle for a liquid-crystal display (LCD), particular attention is being paid to the in-plane switching (IPS) mode of LCD, where pixel electrodes and common (opposite) electrodes are arranged on the same array substrate and liquid crystal is made respond by an electric field generated in parallel to the substrate. So far, two types of array electrode structures are proposed in IPS-LCD. One is the array structure with two electrode layers where pixel electrodes and common electrodes are formed on different conductive layers. In the other array structure they are formed on one conductive layer. Although both electrode structures of IPS-LCD have problems dependent on the process conditions, light leakage observed only in the former structure along the edge of the common electrodes at zero bias is an essentially serious problem. By studying light wave propagation in the IPS-LCD using a two-dimensional finite-difference timedomain method we have found that the light leakage is caused by the tapered side surfaces of the common electrodes.
\end{abstract}

Keywords: liquid-crystal display (LCD), in-plane switching (IPS), finite-difference time-domain method (FDTD), light leakage

\section{Introduction}

In recent years, a display device having a large capacity and a high density has been developed and practiced using liquid-crystal as a display element. In particular, development and commercial production has been widely made of a liquid-crystal display (LCD) element of an active matrix type capable of displaying an image at a high contrast ratio without cross-talk. Further, particular attention is being paid to the so-called In-Plane Switching (IPS) (1) (2) mode of LCD for a wider viewing angle. Fig. 1 shows a schematic diagram of the IPSLCD, where both the pixcel electrode and the common electrode are set on the same glass substrate. On both glass plates the orientation of liquid-crystal molecules is aligned to be nearly parallel to the surface of glass plate. The orientation on each plate is the same as that of the other plate. When the front polarizer is set perpendicular to the rear polarizer, there is no transmission of light at zero bias (normally black). When the voltage is applied to the electrodes, the director of liquid-crystal molecules between the electrodes rotates to align with the electric field parallel to the glass plate, which results in transmission of light.

So far, two types of array electrode structures are proposed in IPS-LCD. One is the array structure with two electrode layers where pixel electrodes and common

\footnotetext{
* Graduate School of Science and Technology, Kobe University 1-1, Rokkodai, Nada-ku, Kobe 657-8501
}

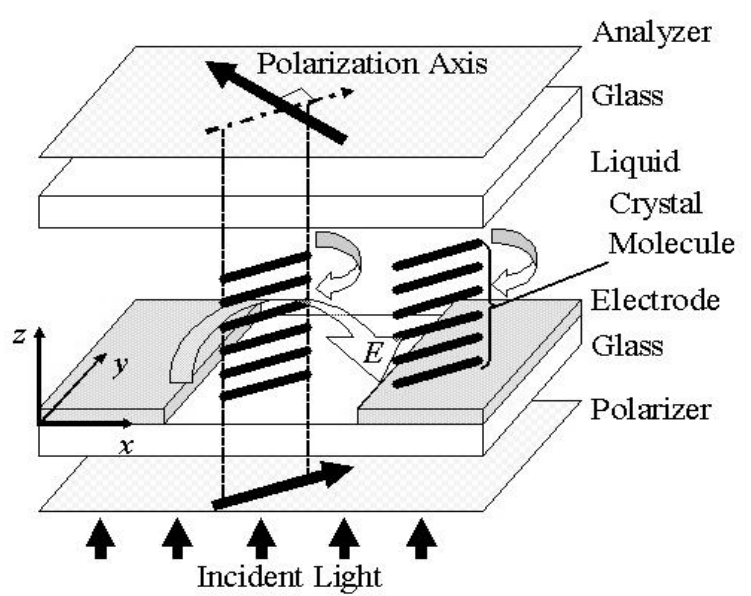

Fig. 1. Schematic diagram of IPS-LCD

electrodes are formed on different conductive layers as shown in Fig. 2 (two-electrode-layers structure). In the other array structure they are formed by processing one conductive layer as shown in Fig. 3 (one-electrode-layer structure).

In the former two-electrode-layers structure, the common electrodes are formed on the same layer as the scanning signal line. They are formed in a tapered shape with a taper angle about $30^{\circ}$ to $60^{\circ}$ with respect to the surface of the substrate to be covered with the insulating film formed by CVD method. In this structure, since the pixel electrodes and the common electrodes 


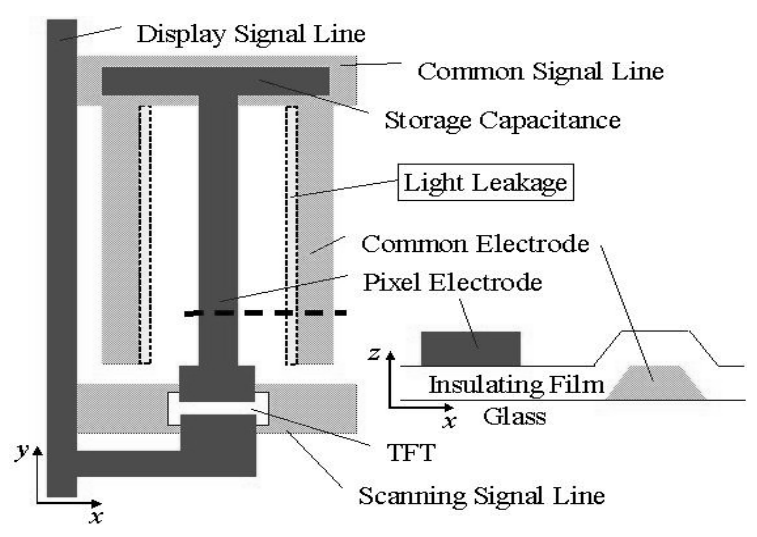

Fig. 2. Two-electrode-layers structure

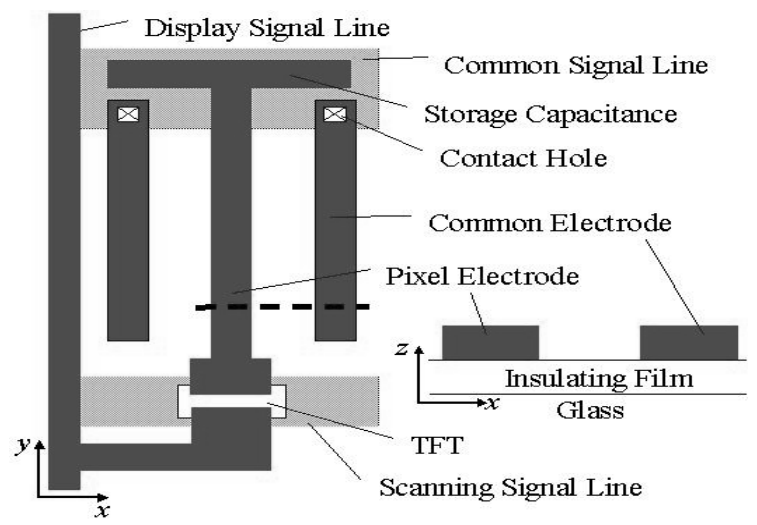

Fig. 3. One-electrode-layer structure

are formed by independent photo engraving process, it is often the case that the distance between the pixel electrode and the common electrode are not uniform due to misalignment patterns during exposure. This nonuniformity deteriorates the display quality of the panel, which is observed as gritty with human eyes.

To improve this problem, one of the authors proposed the one-electrode-layer structure ${ }^{(3)}$ shown in Fig. 3. In this structure, the pixel electrodes and the common electrodes are exposed by using the same photo-mask. Consequently, the distance between the pixel electrode and the common electrode is free from the misalignment and becomes uniform completely. However, in this structure, we often have the defects of contact holes, through which the common electrodes are connected to the common signal lines, and the short circuit defects between the pixel electrodes and the common electrodes.

All of the problems mentioned above are dependent on the process conditions, and related to the yield of liquid crystal devices. However, there is an essentially serious problem in the two-electrode-layers structure, which is the light leakage observed along the edge of the common electrodes at zero bias (under the normally black condition) as shown in Fig. 2. In this paper, in order to evaluate such a leakage phenomenon numerically, light wave propagation in the IPS-LCD is studied using a two-dimensional finite-difference time-domain (FDTD) method.

Conventionally, simulation of liquid crystal optics has been dominated by the application of matrix-type solvers. The $2 \times 2$ Jones matrix $\operatorname{method}^{(4)}$ is used at normal incidence, and the $4 \times 4$ Berreman method ${ }^{(5)}$ is applicable to oblique incidence. However, they are all based on the stratified medium approach and therefore, their application is limited to one-dimensional liquidcrystal structures. In the analysis of IPS-LCD, due to the transverse variation in the liquid-crystal structure, the stratified medium approach is not adequate. Finite-difference time-domain methods represent a powerful approach for direct solution of Maxwell's equations, both in space and time. The FDTD method has been applied to model 2-D light wave propagation in the TN cell ${ }^{(6)}{ }^{(7)}$. In our 2-D simulator newly developed for LCD, the finite element packages for the analysis of liquid-crystal orientation and electrostatic field, and the FDTD package for the 2-D light wave analysis are included.

\section{Analysis}

2.1 Dielectric Permittivity and Refractive Index The liquid-crystal exhibits anisotropy of refractive indices or birefringence, as do optically uniaxial crystals. In the nematic phase, the optical axis coincides with the director of the liquid-crystal. Therefore, the optical dielectric tensor of the liquid-crystal is represented by using two principal refractive indices $n_{o}$ and $n_{e}$, where $n_{o}$ and $n_{e}$ denotes the refractive indices for an ordinary ray and an extraordinary ray, respectively. The birefringence $\Delta n$ corresponds to the difference between the principal refractive indices as $\Delta n=n_{e}-n_{o}$. The local director $\boldsymbol{n}(x, z)=\left(n_{x}, n_{y}, n_{z}\right)$ is dependent upon the tilt $\theta(x, z)$ and twist $\phi(x, z)$ angles through the expression

$$
\boldsymbol{n}(x, z)=(\cos \theta \cos \phi, \cos \theta \sin \phi, \sin \theta)
$$

Then, the relative dielectric tensor $\tilde{\varepsilon}(x, z)$ of the liquidcrystal is represented as

$$
\tilde{\varepsilon}(x, z)=\left[\begin{array}{lll}
\varepsilon_{x x} & \varepsilon_{x y} & \varepsilon_{x z} \\
\varepsilon_{y x} & \varepsilon_{y y} & \varepsilon_{y z} \\
\varepsilon_{z x} & \varepsilon_{z y} & \varepsilon_{z z}
\end{array}\right], \ldots \ldots \ldots \ldots \ldots
$$

where each component of $\tilde{\varepsilon}$ is given by

$$
\varepsilon_{i j}=n_{o}^{2} \delta_{i j}+\left(n_{e}^{2}-n_{o}^{2}\right) n_{i} n_{j} \quad(i, j=x, y, z),
$$$$
\text { ................ }
$$

where $\delta_{i j}$ is Kronecker's delta.

On the other hand, for the electrostatic dielectric permittivity, its component is given by

$$
\varepsilon_{i j}=\varepsilon_{\perp} \delta_{i j}+\left(\varepsilon_{\|}-\varepsilon_{\perp}\right) n_{i} n_{j}, \cdots \cdots \ldots \ldots \ldots
$$

where $\varepsilon_{\|}$and $\varepsilon_{\perp}$ denote the relative dielectric permittivity in the directions parallel and perpendicular to the director, respectively.

In an IPS cell structure, the molecules at the boundaries are strongly coupled (anchored) to the surfaces and tilted several degrees by rubbing process. The tilt angle on the surface is called the pretilt angle. However, the liquid-crystal molecules except at boundaries 
are easily affected by an external electric field. Therefore, the electrostatic field problem and the liquid crystal orientation problem are coupled together and solved self-consistently. We will use a finite element method (FEM) as a numerical analysis method of them.

2.2 Self-Consistent Analysis of Liquid-Crystal Orientation and Electrostatic Field by FEM The fundamental expression for the free-energy density for nematics $f_{\text {elas }}$ is given by

$$
\begin{aligned}
f_{\text {elas }}= & \frac{1}{2} k_{11}(\nabla \cdot \boldsymbol{n})^{2}+\frac{1}{2} k_{22}\{\boldsymbol{n} \cdot(\nabla \times \boldsymbol{n})\}^{2} \\
& +\frac{1}{2} k_{33}\{\boldsymbol{n} \times(\nabla \times \boldsymbol{n})\}^{2} . \cdots \cdots \cdots
\end{aligned}
$$

The above free energy is the one for infinitesimal deformation of liquid-crystal relative to that in the state of uniform orientation. The three elastic constants $k_{11}$, $k_{22}$, and $k_{33}$ are called the splay, twist, and bend constants, respectively ${ }^{(8)}{ }^{(9)}$. On the other hand, the energy density $f_{\text {elec }}$ that the liquid crystal receives from electric field is given by

$$
f_{\text {elec }}=-\frac{1}{2} \varepsilon_{0} \Delta \varepsilon(\boldsymbol{n} \cdot \boldsymbol{E})^{2}, \quad \Delta \varepsilon=\varepsilon_{\|}-\varepsilon_{\perp} . \cdots
$$

The total energy $f$ given by the sum of Eq. (5) and Eq. (6), $f=f_{\text {elas }}+f_{\text {elec }}$, is used as an energy functional for the FEM analysis of the liquid-crystal orientation. From the standard finite element discretization, a forth-order nonlinear matrix equation in terms of director vectors is obtained and solved with the linear matrix equation of electrostatic potential simultaneously.

To solve the nonlinear equations of director vectors numerically, it is linearized first by using the incremental analysis method. Then, the distributions of director orientation are found by the iterative procedure.

The procedure to obtain self-consistent solutions of liquid-crystal orientation and electrostatic field for a certain applied voltage is summarized in the flow chart of

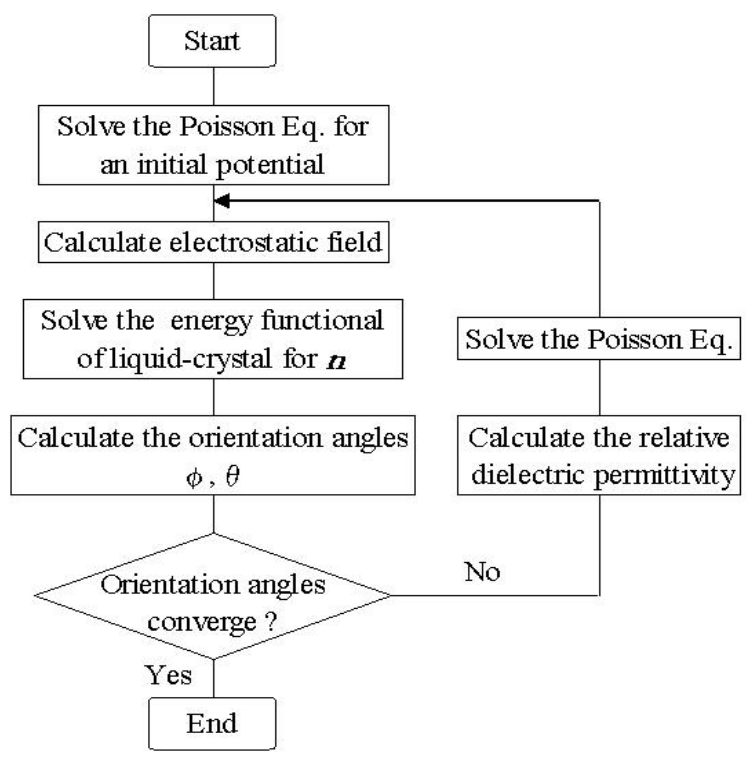

Fig. 4. Flow chart of the self-consistent calculation
Fig. 4. In the practical implementation, the voltage is gradually increased from $0 \mathrm{~V}$ to the applied bias voltage with a small voltage step, and at every voltage step, the self-consistent solutions are calculated according to the flow chart shown in Fig. 4.

2.3 Light Wave Analysis by FDTD Method Fig. 5 shows an IPS-LCD model used in the FDTD analysis. In liquid crystal, electromagnetic field is governed by Maxwell's equations.

The computational space is divided by the twodimensional Yee cells ${ }^{(10)}$, where all six field-components are present as shown in Fig. 6. It is further assumed that $\boldsymbol{E}$ is available only at integer values of $\Delta t$, while $\boldsymbol{H}$ is available a half time step away. Dielectric displacement components are located at the same grid positions as the electric field components. When the function $A(x, z, t)$ is evaluated at a point $(i \Delta x, j \Delta z, n \Delta t)$, it is written $\left.A\right|_{i, j} ^{n}$.

In FDTD method, each of the component is obtained by applying a central difference to the Maxwell's equations. As an example, the $y$ components of the fields in the liquid-crystal material are updated through

$$
\begin{aligned}
\left.D_{y}\right|_{i, j} ^{n+1}= & \left.D_{y}\right|_{i, j} ^{n}+\Delta t\left\{\frac{\left.H_{x}\right|_{i, j+1} ^{n+\frac{1}{2}}-\left.H_{x}\right|_{i, j} ^{n+\frac{1}{2}}}{\Delta z}\right. \\
& \left.-\frac{\left.H_{z}\right|_{i+1, j} ^{n+\frac{1}{2}}-\left.H_{z}\right|_{i, j} ^{n+\frac{1}{2}}}{\Delta x}\right\} \ldots \ldots \ldots \\
\left.H_{y}\right|_{i, j} ^{n+\frac{1}{2}}= & \left.H_{y}\right|_{i, j} ^{n-\frac{1}{2}}+\Delta t\left\{\frac{\left.E_{z}\right|_{i, j} ^{n}-\left.E_{z}\right|_{i-1, j} ^{n}}{\Delta x}\right. \\
& \left.-\frac{\left.E_{x}\right|_{i, j} ^{n}-\left.E_{x}\right|_{i, j-1} ^{n}}{\Delta z}\right\} \ldots \ldots \ldots .
\end{aligned}
$$

where the electric field is deduced from the dielectric displacement, for example

$$
\begin{aligned}
\left.E_{y}\right|_{i, j} ^{n}= & \frac{1}{\varepsilon_{0} \operatorname{det}[\tilde{\varepsilon}]}\left[\left.\left(\varepsilon_{z x} \varepsilon_{y z}-\varepsilon_{y x} \varepsilon_{z z}\right) D_{x}\right|_{i+\frac{1}{2}, j} ^{n}\right. \\
& +\left.\left(\varepsilon_{x x} \varepsilon_{z z}-\varepsilon_{x z} \varepsilon_{z x}\right) D_{y}\right|_{i, j} ^{n} \\
& \left.+\left.\left(\varepsilon_{x z} \varepsilon_{y x}-\varepsilon_{x x} \varepsilon_{y z}\right) D_{z}\right|_{i, j+\frac{1}{2}} ^{n}\right], \cdots
\end{aligned}
$$

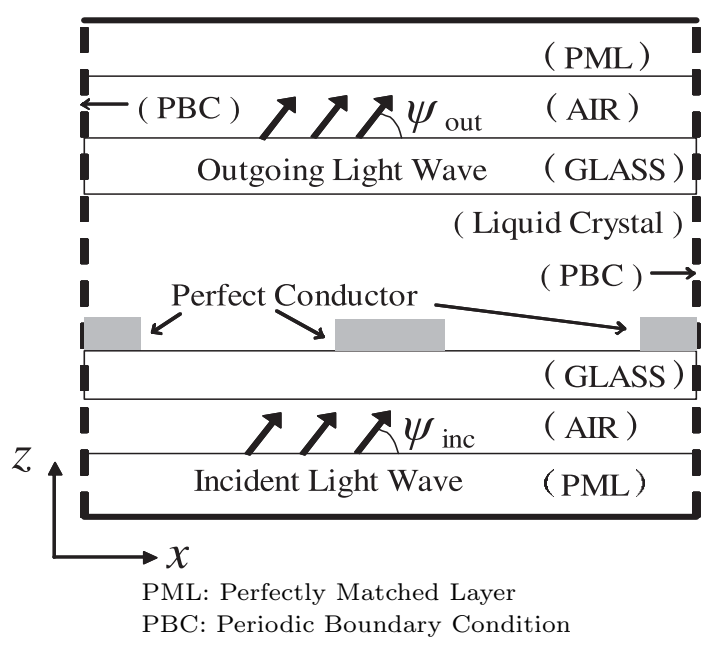

Fig. 5. IPS-LCD model used in the FDTD analysis 


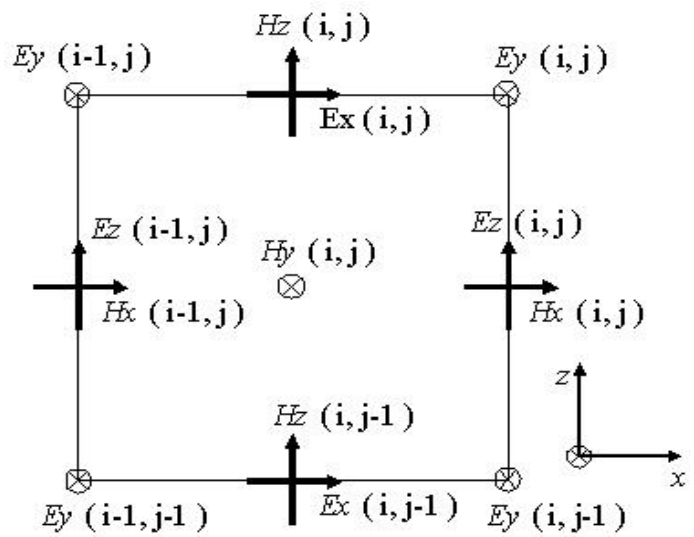

Fig. 6. Two-dimensional Yee space lattice

where $\operatorname{det}[\tilde{\varepsilon}]$ denotes a determinant of $\tilde{\varepsilon}$. However, since the dielectric displacement components are not available at the positions $(i+1 / 2, j)$ and $(i, j+1 / 2)$, they are approximated as

$$
\begin{aligned}
& \left.D_{x}\right|_{i+\frac{1}{2}, j} ^{n}=\frac{1}{2}\left[\left.D_{x}\right|_{i+1, j} ^{n}+\left.D_{x}\right|_{i, j} ^{n}\right] \ldots \ldots \ldots \ldots \\
& \left.D_{z}\right|_{i, j+\frac{1}{2}} ^{n}=\frac{1}{2}\left[\left.D_{z}\right|_{i, j+1} ^{n}+\left.D_{z}\right|_{i, j} ^{n}\right] \ldots \ldots \ldots
\end{aligned}
$$

Thus, the electric field values are obtained when all three dielectric displacement components have been time-advanced, and they are subsequently used when time-advancing the magnetic field as given by Eq. (8).

As shown in Fig. 5, the computational space is truncated with the uniaxial perfectly matched layers $(\mathrm{PML})^{(11)}$ in the normal direction. The PML is an artificial absorbing material characterized by anisotropic electric conductivity and magnetic resistivity. The PML provides a reflectionless interface to the radiation for all possible angles of incidence, and suppresses any radiation leaving the upper and lower faces of the computational space. The transverse termination is provided by imposing periodic boundary conditions (PBC). In the practical implementation of the $\mathrm{PBC}$, the sin-cos dual plane-wave excitation (dual-time source) is developed in ${ }^{(12)}$, where the enforcement of the phase shift due to the periodicity is made straightforward.

The incident plane wave is launched in the FDTD method grid by a total field/scattered field formulation as suggested in ${ }^{(13)}$. In this paper, the incident field is given on the lower surface of the lower glass substrate. Here, it should be noted that numerical waves in a twodimensional Yee space lattice have a propagation velocity that is dependent upon the direction of wave propagation. Such a numerical dispersion is a typical numerical error in FDTD method, and it is expected to be suppressed by reducing the lattice space and time increments. However, in the expression for impinging plane wave, we have to use the numerical phase velocities derived from the numerical dispersion relation.

\section{Numerical Results}

The geometry of the two-electrode-layers structure, that we analyze here, is illustrated in Fig. 7 . It is comprised by a center pixel electrode, half of the right

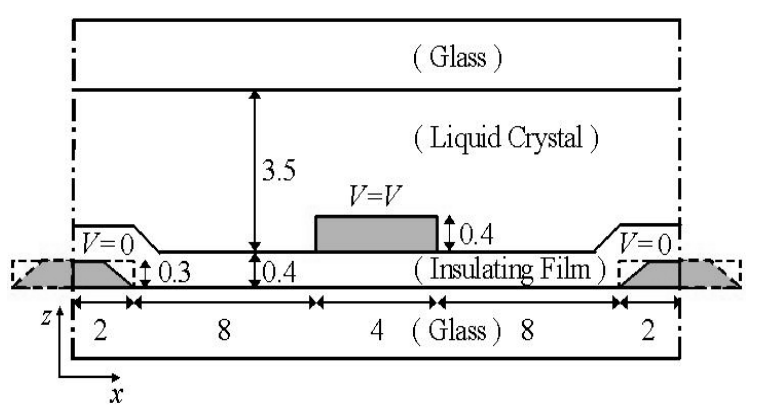

Fig. 7. Geometry of the two-electrode-layers structure (unit: $\mu \mathrm{m}$ )

Table 1. Liquid crystal material parameters used in the calculation

\begin{tabular}{llr}
\hline \multicolumn{1}{c}{ Parameter } & \multicolumn{1}{c}{ Value } \\
\hline \hline Elastic constant & $k_{11}$ & $8.7[\mathrm{pN}]$ \\
& $k_{22}$ & $5.1[\mathrm{pN}]$ \\
& $k_{33}$ & $14.4[\mathrm{pN}]$ \\
\hline Static relative dielectric constant & $\varepsilon_{\|}$ & 13.9 \\
& $\varepsilon_{\perp}$ & 4.0 \\
\hline Optical refractive index & $n_{e}(650 \mathrm{~nm})$ & 1.56 \\
& $n_{o}(650 \mathrm{~nm})$ & 1.47 \\
\hline
\end{tabular}

common electrode, and half of the left common electrode. Periodic repetition of the block shown in Fig. 7 provides an IPS display. In case of the one-electrodelayer structure, the insulating film is removed and the tapered common electrodes on the glass substrate are replaced by the rectangular shaped electrodes. The liquidcrystal material parameters used in the calculation are summarized in Table 1. In case of the one-electrodelayer structure, the anchoring tilt and twist angles of molecules are $3^{\circ}$ and $70^{\circ}$, respectively, that are given on the upper and lower glass substrates and the electrodes except side surfaces. In case of the two-electrode-layers structure, the anchoring tilt angle is also set $3^{\circ}$ on the tapered surface which is inclined with $45^{\circ}$ with respect to the surface of the substrate. The polarizers are arranged in normally black configuration. Material of the glass substrate and the insulating film are assumed to be $\mathrm{SiO}_{2}$, and their refractive indices are 1.5 .

Fig. 8 shows the distribution of light transmittance in the normal direction for the one-electrode-layer structure at $0 \mathrm{~V}$. In this calculation, incident light is provided by simultaneous illumination of many plane waves with the incident angles $\psi_{\text {inc }}$ from $10^{\circ}$ to $170^{\circ}$ at angular intervals of $2^{\circ}$ to simulate the practical backlight. Accordingly, the transmitted light in the normal direction is defined as the total transmitted light with outgoing angles from $89^{\circ}$ to $91^{\circ}$. The normal transmittance in Fig. 8 is the ratio of the normal outgoing light defined above to the normal incident one at each point on the $x$-axis. The normal transmittance distributions of the two-electrode-layers structure are shown in Fig. 9 for rectangular common electrodes and in Fig. 10 for tapered common electrodes, respectively. No great difference of normal transmittance is found in Fig. 8 and Fig. 9 , while the transmittance at the edge of the tapered common electrodes are found higher than those of other structures in Fig. 10. To study the origin of such a light 


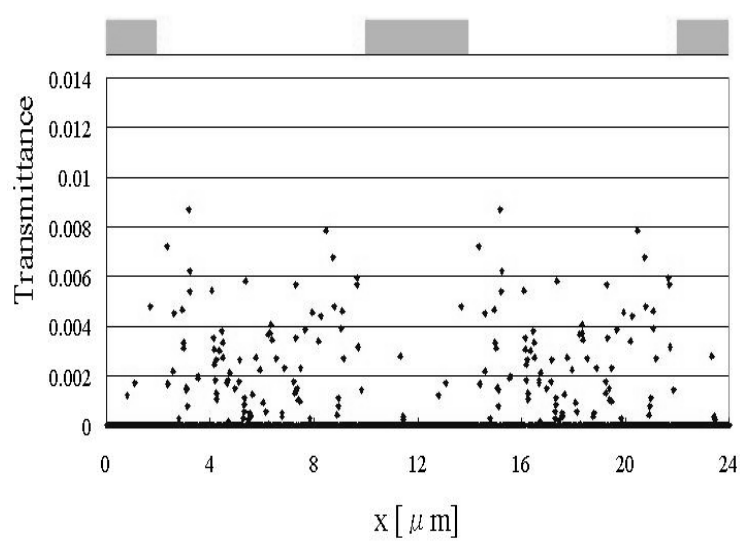

Fig. 8. Normal light transmittance of the oneelectrode-layer structure at $0 \mathrm{~V}$

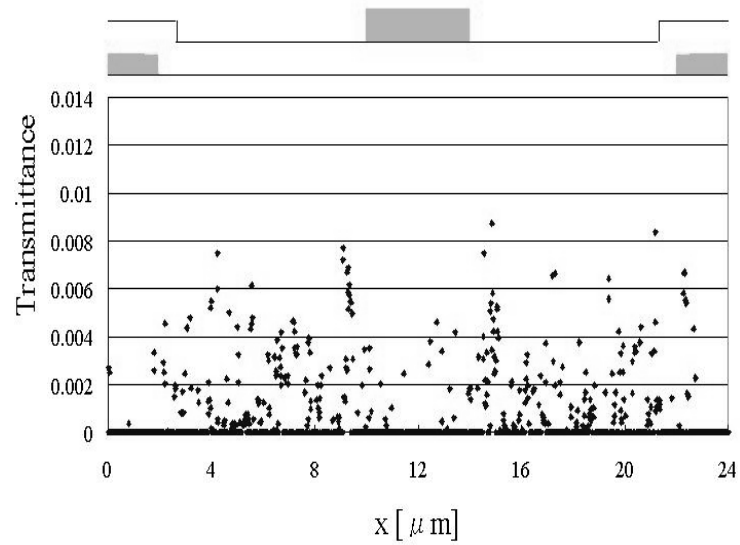

Fig. 9. Normal light transmittance of the twoelectrode-layers structure with rectangular shaped common electrodes at $0 \mathrm{~V}$

leakage, the liquid-crystal orientation are calculated as shown in Fig. 11 and Fig. 12 for the two structures, where the director profile is projected to the $x-z$ plane. It is found that in the two-electrode-layers structure the liquid-crystal molecules are strongly affected by the anchoring of the tapered surface at zero bias and nonuniform profile extends in relatively wide area, while the almost homogeneous orientation exists in the cell for the one-electrode-layer structure. Further, due to the asymmetrical distributions of liquid-crystal orientation at the right side and the left side of tapered electrodes, the slightly different amounts of peek transmittance are found around $x=3 \mu m$ and $x=21 \mu m$. The isolated point around at $x=15 \mu \mathrm{m}$ in Fig. 10 is puzzling. It may be concerned with diffraction at the corner of perfect rectangular electrode used in this calculation. We need further study on this issue.

Fig. 13 shows the normal light transmittance of the two structures as a function of applied voltage. In Fig. 13 the transmittances for both structures are almost the same at low bias. It means that although the light leakage observed in the two-electrode-layers structure at low bias causes a textured feeling for human eyes which is an essentially serious problem, it gives no effects

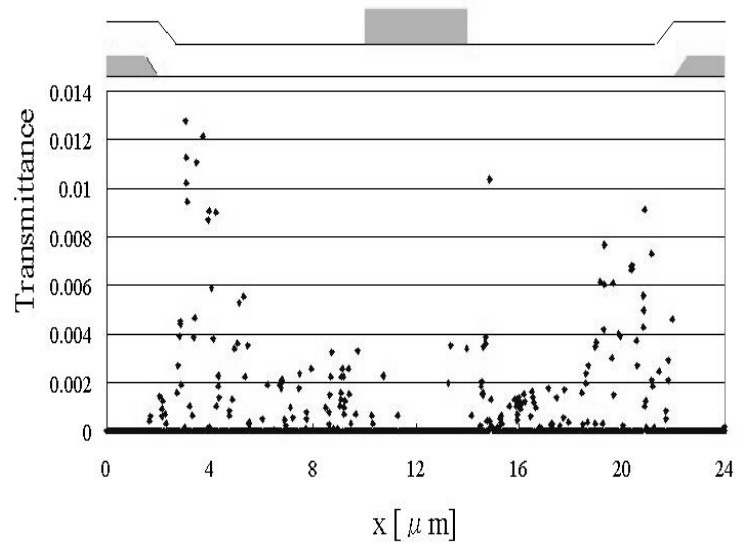

Fig. 10. Normal light transmittance of the twoelectrode-layers structure with tapered common electrodes at $0 \mathrm{~V}$

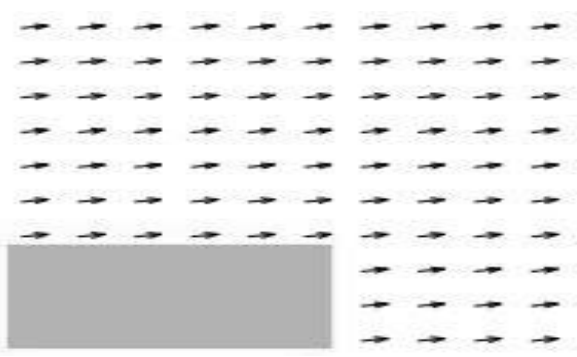

Fig. 11. Orientation distribution of the oneelectrode-layer structure at $0 \mathrm{~V}$

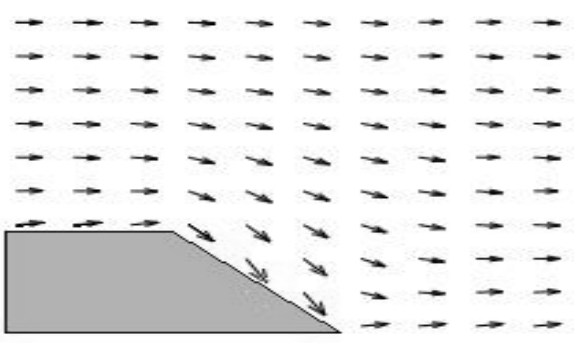

Fig. 12. Orientation distribution of the twoelectrode-layers structure with taper at $0 \mathrm{~V}$

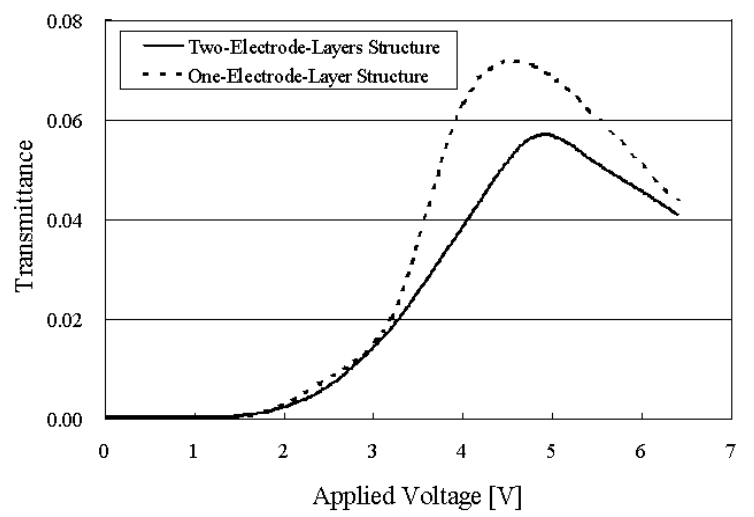

Fig. 13. Normal light transmittance as a function of applied voltage

on averaged brightness. Here, note that the transmittance in Fig. 13 is defined for a whole analysis region. The Maximum light transmittance of $7.2 \%$ at $4.6 \mathrm{~V}$ and 
the contrast ratio 170 of the one-electrode-layer structure are better than $5.7 \%$ at $4.9 \mathrm{~V}$ and 147 of the twoelectrode-layers structure, respectively. Since the director of liquid-crystal molecules align with the electric field at high applied voltage, the influence due to the anchoring observed at zero bias may be small at this voltage. The strength of electric field becomes weaker in the twoelectrode-layers structure due to the insulating film between the electrodes, which must be responsible for the deterioration of light transmittance of this structure.

\section{Conclusion}

We have analysed the orientation of liquid-crystal molecules and the light wave propagation characteristics to evaluate the array electrode structures of IPS-LCD. Although the array electrode structures of IPS-LCD have problems dependent on the process conditions, the light leakage observed only in the two-electrode-layers structure along the edge of the common electrodes at zero bias is an essentially serious problem. By studying light wave propagation in the IPS-LCD using a two-dimensional finite-difference time-domain method we have found that the light leakage is caused by the tapered side surfaces of the common electrodes in the two-electrode-layers structure. The orientation of liquidcrystal molecules is strongly distorted by the anchoring of the tapered surface at zero bias and nonuniform profile extends in relatively wide area. We conclude that the nonuniformity is responsible for the light leakage in the two-electrode-layers structure.

(Manuscript received Feb. 16, 2004, revised Aug. 16, 2004)

\section{References}

(1) M. Oh-e, M. Ohta, S. Aratani, and K. Kondo: "Principles and characteristics of electro-optical behaviour with In-Plane Switching mode", Proc. of the 15th International Display Reserch Conference (Asian Display '95), pp.577-580 (1995)

(2) M. Ohta, M. Oh-e, and K. Kondo: "Development of SuperTFT-LCDs with In-Plane Switching display mode", Proc. of the 15th International Display Reserch Conference (Asian Display '95), pp.707-710 (1995)

( 3 ) H. Kawano and T, Kamimura: "Array Substrate for Liquid Crystal Display Element", USP, US 6,337,726 BI (2002-1)

(4) R.C. Jones: "A new calculus for the treatment of optical systems I. description and discussion of the calculus", J. Opt. Soc. Am. Vol.3, pp.488-493 (1941)

( 5 ) D.W. Berreman: "Optics in stratified and anisotropic media $4 \times 4$-matrix formulation", J. Opt. Soc. Am. Vol.62, pp.502510 (1972)

(6) E.E. Kriezis and S.J. Elston: "Finite-difference time domain metod for light wave propagation within liquid crystal devices", Opt. Commun., Vol.165, pp.99-105 (1999)

( 7 ) E.E. Kriezis and S.J. Elston: "Light wave propagation in liquid crystal displays by the 2-D finite-difference time-domain method", Opt. Commun., Vol.177, pp.69-77 (2000)

(8) F.C. Frank: "On the Theory of Liquid Crystal", Discuss. Faraday Soc., Vol.25, pp.19-28 (1958)

(9) C.Z. van Doorn: "Dynamic behavior of twisted nematic liquidcrystal layers in switched fields", J. Appl. Phys. Vol.46, pp.3738-3745 (1975)

(10) K. Yee: "Numerical solution of initial boundary value problems involving Maxwell's equations in isotropic media", IEEE Trans. Antennas Propagat., Vol.14, pp.302-307 (1966)
(11) S.D. Gedney: "Ananisotropic perfectly matched layerabsorbing medium for the truncation of FDTD lattices", IEEE Trans. Antennas Propagat., Vol.44, pp.1630-1639 (1996)

(12) P.H. Harms, R. Mittra, and W. Ko: "Implementation of the periodic boundary condition in the finite-difference timedomain algorithm for FSS structures", IEEE Trans. Antennas Propagat., Vol.42, pp.1317-1324 (1994)

(13) A. Toflove and S.C. Hagness: Computational Electrodynamics, The finite-difference time-domain method, Second Ed., pp.67-233, Artech House Publishers (2000)

Hideo Kawano (Student Member) was born in Osaka,

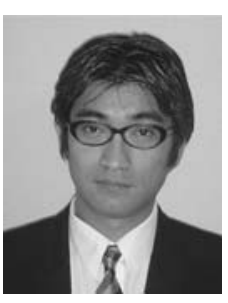
Japan, in 1965. He received the B.E degree from Osaka Prefecture University, Japan, in 1990. He joined Electronic Engineering Laboratories of Toshiba Inc., Yokohama, Japan, in 1990, where he has been engaged in development of liquid-crystal display. He is currently pursuing the Ph.D. degree in graduate school of science and technology, Kobe University, Japan.

Atsushi Matsumoto (Non-member) was born in Kyoto,

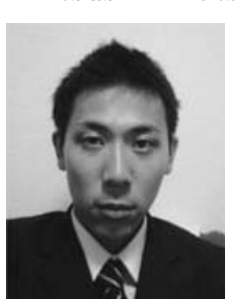
Japan. He received the B.E. degree from Kobe University, Japan, in 2002, and is currently working toward his master's degree in graduate school of science and technology, Kobe University, Japan.

Johji Hirano (Non-member) was born in Japan. He re-

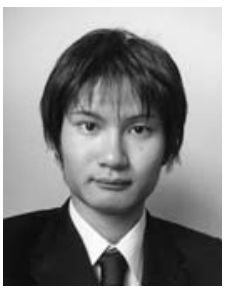
ceived the B.E. degree from Osaka Institute of Technology, Japan, in 2002, and is currently working toward his master's degree in graduate school of science and technology, Kobe University, Japan.

Tanroku Miyoshi (Non-member) received the B.S. in elec-

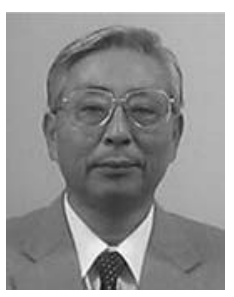
trical engineering from the University of Tokyo, Tokyo, Japan, in 1967, and the M.S. and Ph.D. degree in electronics engineering from the University of Tokyo, Tokyo, Japan in 1969 and 1972, respectively. In 1972 he was appointed Lecture, and from 1974 to 1987, he was an Associate Professor in the Department of Electronics Engineering, Kobe University, Kobe, Japan, where he is presently a Professor. He has been engaged in research of electromagnetic-wave theory, microwave integrated circuits, and light-wave electronics. His current research includes quantum transport modeling in nanostructures. In 1976 he was a Visiting Scholar at McGill University, Montreal, Que., Canada. From 1982 to 1984, he was a Visiting Scientist at Bell Laboratories, Holmdel, NJ. He is a member of the IEEE and the Japan Society of Applied Physics. He received a Yonezawa Award in 1974 and an Outstanding Book Award in 1977 both from the Institute of Electronics, Information and Communication Engineers in Japan. 